\title{
Correction to: Attitudes of physicians from 10 European countries on adherence and how treatment modalities in ABSSSI affect adherence: results from a Delphi survey
}

\author{
Tom Stargardt ${ }^{1} \cdot$ Christian Eckmann $^{2} \cdot$ Emilio Bouza $^{3,4,5,6} \cdot$ Gian Maria Rossolini $^{7,8} \cdot$ Paolo Antonio Grossi ${ }^{9}$
}

Published online: 12 July 2018

(C) Springer-Verlag GmbH Germany, part of Springer Nature 2018

\section{Correction to: European Journal of Clinical Microbiology \& Infectious Diseases https://doi.org/10.1007/s10096-018-3264-0}

In the originally published article, the name of the last author was not correct. The name is Paolo Antonio Grossi, which is correctly shown above.

The original article has been corrected.

The online version of the original article can be found at https://doi.org/ 10.1007/s10096-018-3264-0

Tom Stargardt

tom.stargardt@uni-hamburg.de

1 Hamburg Center for Health Economics, University of Hamburg, Esplanade 36, 20354 Hamburg, Germany

2 Department of General, Visceral, and Thoracic Surgery, Klinikum Peine, Academic Hospital of Medical University Hannover, Hannover, Germany

3 Department of Clinical Microbiology and Infectious Diseases, Hospital General Universitario Gregorio Marañón, Madrid, Spain

4 Instituto de Investigación Sanitaria Gregorio Marañón, Madrid, Spain
5 Medicine Department, School of Medicine, Universidad Complutense de Madrid, Madrid, Spain

6 CIBER Enfermedades Respiratorias-CIBERES (CB06/06/0058), Madrid, Spain

7 Department of Experimental and Clinical Medicine, University of Florence, Florence, Italy

8 Microbiology and Virology Unit, Florence Careggi University Hospital, Florence, Italy

9 Department ofMedicine and Surgery, Section of Infectious Diseases, University of Insubria, Varese, Italy 